\title{
SARC-F as a case-finding tool for sarcopenia according to the EWGSOP2. National validation and comparison with other diagnostic standards
}

\author{
Karolina Piotrowicz ${ }^{1,2}$. Anna Głuszewska ${ }^{1,2}$. Joanna Czesak ${ }^{2,3}$. Małgorzata Fedyk-Łukasik ${ }^{1,2}$. Ewa Klimek ${ }^{1,2}$. \\ Dolores Sánchez-Rodríguez ${ }^{4,5} \cdot$ Anna Skalska ${ }^{1,2} \cdot$ Barbara Gryglewska $^{1,2} \cdot$ Tomasz Grodzicki $^{1,2} \cdot$ Jerzy Gąsowski $^{1,2}(1)$
}

Received: 22 July 2020 / Accepted: 22 December 2020 / Published online: 28 January 2021

(c) The Author(s) 2021

\begin{abstract}
Background Sarcopenia is a potentially reversible condition, which requires proper screening and diagnosis.

Aims To validate a Polish version of sarcopenia screening questionnaire (SARC-F), and assess its clinical performance. Methods Cross-sectional validation study in community-dwelling subjects $\geq 65$ years of age. Diagnosis of sarcopenia was based on the 2018 2nd European Working Group on Sarcopenia in Older People (EWGSOP2) consensus. Hand grip and 4-m gait speed were measured, and the Polish version of SARC-F was administered.

Results The mean (SD) age of 73 participants (21.9\% men) was 77.8 (7.3) years. Seventeen participants (23.3\%) fulfilled the EWGSOP2 criteria of sarcopenia, and 9 (12.3\%) criteria for severe sarcopenia. Fourteen (19.2\%) participants fulfilled the SARC-F criteria for clinical suspicion of sarcopenia. The Cronbach's alpha coefficient for internal was 0.84 . With EWGSOP2 sarcopenia as a gold standard, the sensitivity of SARC-F was 35.3\% (95\% CI 14.2-61.7, $p=0.33$ ), specificity was 85.7\% (95\% CI 73.8-93.6, $p<0.0001$ ). The corresponding positive and negative predictive values were $42.9 \%(p=0.79)$ and $81.4 \%(p<0.0001)$, respectively. The probability of false-positive result was $14.3 \%$ (95\% CI 6.4-26.2, $p<0.0001$ ) and the probability of false-negative result was $64.7 \%$ (95\% CI 38.3-85.8, $p=0.33$ ). Overall the predictive power of SARC-F was low (c-statistic 0.64).

Discussion SARC-F is currently recommended for sarcopenia case finding in general population of older adults. However, its sensitivity is low, despite high specificity.

Conclusions At present SARC-F is better suited to rule out sarcopenia then to case-finding. Further refinement of screening for sarcopenia with the use of SARC-F seems needed.
\end{abstract}

Keywords Sarcopenia $\cdot$ Screening $\cdot$ SARC-F $\cdot$ DXA $\cdot$ EWGSOP2

SARC-F is a screening tool for sarcopenia. We found it's Polish translation intra, and inter-rater reproducible, with low sensitivity and good specificity vs. the EWGSOP2 definition of sarcopenia, with DXA quantification of muscle.

Supplementary Information The online version contains supplementary material available at https://doi.org/10.1007/s4052 0-020-01782-y.

Jerzy Gąsowski

jerzy.gasowski@uj.edu.pl

Extended author information available on the last page of the article

\section{Introduction}

Sarcopenia is a frequent, age-related muscle wasting, that results in impaired skeletal muscle performance. Its prevalence has been estimated at 9-40\% among older persons [1]. Sarcopenia has been linked to higher morbidity and mortality [2], increasing the need for support in the activities of daily living or institutionalization, and diminished quality of life [3, 4]. Sarcopenia is also responsible for high care-related burden, including burden to the family and the society $[5,6]$. Sarcopenia is amenable to therapy, mainly rehabilitation and proper nutrition [7, 8]. However, protocols to assess sarcopenia are required, with sufficient performance in the case-finding and the confirmation stages of the diagnosis [9]. 
SARC-F is a simple, easy-to-use, 5-item sarcopenia screening questionnaire [10]. Since then, SARC-F has been translated and validated for use in different languages [11-16], various clinical settings [11, 13, 14, 17], and against various gold-standard diagnostic modalities then current [18, 19]. In 2018, SARC-F has been incorporated, as a sarcopenia case-finding tool, in the European Working Group on Sarcopenia in Older People 2 (EWGSOP2) diagnostic algorithm for sarcopenia [6].

However, the diagnostic performance of the test varies across studies [20]. As an example, the sensitivity of the test varies considerably across studies, with values ranging from 3.9 to $95.4 \%[21,22]$.

Our aim, was to standardize the translation and validate the clinical performance of the Polish version, included in the on-line appendix, of the SARC-F as a case-finding tool against an array of objective diagnostic criteria for sarcopenia, including dual-energy X-ray absorptiometry (DXA)based EWGSOP2.

\section{Methods}

\section{Study}

The study was approved by the Jagiellonian University Bioethics Committee (KBET no.: 1072.6120.71.2018). All participants gave written informed consent. The study was performed cross-sectionally between August 2018 and March 2019, in line with the call by the Special Interest Group (SIG) for Sarcopenia of the European Geriatric Medicine Society (EuGMS) for action to improve the screening and the diagnosis of sarcopenia [23].

\section{SARC-F questionnaire and other sarcopenia screening modalities}

We performed a two-step cultural and clinical validation of SARC-F questionnaire in subjects $\geq 65$ years of age. The validation protocol was based on the original validation procedure of the English SARC-F [23]. In brief, the questionnaire was translated from English to Polish by the two Polish geriatric researchers fluent in general and medical English (KP, AS). The final version of the Polish SARC-F was back-translated to English by a Polish-English bilingual native-speaker certified in interpreting between both languages. The back-translation was then sent to Prof. John Morley for the formal approval. The threshold for sarcopenia case-finding has been established at 4 points [10].

The original SARC-F questionnaire together with the Polish translation is included in Appendix 1 [10]. In addition to the SARC-F, we used the SARC-CalF which adds calf circumference to the SARC-F components [24]. We also used mid-arm circumference and calf circumference as separate screening instruments. For the calf and mid-arm circumference, we used population-specific cut-off values based on Youden's Index. For the calculation SARC-CalF, on a scale from 0 to 20, we used the Youden Index-based population-specific cut off for calf circumference $(<32 \mathrm{~cm})$ with a weight of 10 . The cut-off for SARC-CalF was $\geq 11$. We also used index by Ishii et al. [25].

\section{Study population}

Information about the study was sent to the local seniors' organizations (e.g. universities of the third age, senior clubs) and posted in the outpatient and inpatient geriatric clinics of the University Hospital in Kraków. Consecutive communitydwelling people aged 65 years and more were encouraged to participate in the project.

\section{Measures}

Body composition was assessed by the Lunar iDXA dualenergy X-ray absorptiometry (DXA) equipment (GE Healthcare, Chicago, Il, USA). Appendicular lean body mass was calculated as a sum of lean mass of both upper and lower limbs, and expressed and further analyzed as Appendicular Skeletal Muscle Mass (ASM, kg), ASM adjusted for subject's height $\left(\mathrm{ASM} / \mathrm{h}^{2}, \mathrm{~kg} / \mathrm{m}^{2}\right)$ and ASM adjusted for subject's body mass index (BMI) (ASM/BMI) [6] .

Muscle strength $(\mathrm{kg})$ was assessed with a Saehan handheld dynamometer SH 5001 (Seahan Corporation, Masan, South Korea) according to the American Society of Hand Therapists (ASHT) recommendations [26]. Handgrip strength of both hands was assessed three times, and the highest value for either hand was recorded.

Gait speed $(\mathrm{m} / \mathrm{s})$ was measured three times, over a distance of $4 \mathrm{~m}$ with subjects walking at their usual speed, with walking aids if needed [27]. The first attempt was considered as an instructive example, and the highest value of the second or third trials was used.

Additionally, we performed standard Timed-Up and Go (TUG) Test and the Short Physical Performance Battery (SPPB) $[28,29]$. The functional measurements were performed by a study physiotherapist (JC).

The subjects were interviewed by one of the two trained raters (KP, AG). To assess their cognitive performance we used Polish version of the Montreal Cognitive Assessment and 15-point Geriatric Depression Scale [30, 31], functional status the Activities of Daily Living and Instrumental Activities of Daily Living Scales and activity the SevenDay Physical Activity Recall questionnaire [32, 33]. Physical frailty was assessed according to criteria by Fried et al. and Rockwood et al. [34, 35], the nutritional status with the Mini-Nutritional Assessment [36], and quality of life 
with the EuroQol-5D-5L questionnaire [37]. Sociodemographic characteristics and information about medications and comorbidities were collected. Based on the available data, the Charlson Index and total weekly energy expenditure were calculated for each subject $[33,38]$.

Anthropometric measures were performed in accordance with the Centres for Disease Control and Prevention (CDC) guidelines [39], and included: height $(\mathrm{cm})$, weight $(\mathrm{kg})$, waist and hip circumference $(\mathrm{cm})$, calf circumference $(\mathrm{CC}$, $\mathrm{cmf}$ ) and midarm circumference (MAC, $\mathrm{cm}$ ); Body Mass Index $\left(\mathrm{kg} / \mathrm{m}^{2}\right)$ and Waist-Hip Ratio (WHR) was calculated, respectively.

\section{Assessment of sarcopenia}

Sarcopenia was diagnosed according to four definitions including: the European Working Group on Sarcopenia in Older People 2 (EWGSOP2, 2018) consensus (all sarcopenia, not limited to severe sarcopenia)[6], the Foundation for the National Institutes of Health (FNIH, 2014), based on weakness and low muscle mass [40], the International Working Group on Sarcopenia (IWGS, 2011) [41], and the Society of Sarcopenia, Cachexia and Wasting Disorders (SSCWD, 2011) criteria [42]. For the purpose of the presented analysis, we used the values of AMS adjusted to height $\left(\mathrm{ASM} / \mathrm{h}^{2}\right)$ of $\leq 6.0 \mathrm{~kg} / \mathrm{m} 2$ for women and $\leq 7.0 \mathrm{~kg} / \mathrm{m}^{2}$ for men, respectively, as proposed by the EWGSOP2 consensus paper [6]. The operational criteria used for sarcopenia diagnosis are summarized and presented in Appendix 2.

\section{Statistical analyses}

The data management and the statistical analyses were performed with SAS 9.4 (SAS Institute Inc., Cary, NC, USA). The continuous variables were compared with standard normal Z-test or Wilcoxon's test, normally and non-normally distributed variables, respectively. The proportions were compared with chi-square test. To assess the coherence of the Polish version of the SARC-F inventory, we calculated Cronbach's alpha coefficient. Further, for each objective gold-standard diagnosis of sarcopenia as a binary outcome, with SARC-F as an ordinal explanatory variable on a scale from zero to ten, we fitted a logistic regression model based on which we obtained the Receiver Operating Characteristics (ROC) curve with the Area Under the Curve (AUC) as a measure of diagnostic performance. Using the approach described by Youden et al. [43] we obtained the population-specific cut off values for SARC-F. Further, based on the cut-off of 4 proposed by the EWGSOP2, and the calculated population-specific cut-off, we calculated the sensitivity, specificity, positive predictive value, negative predictive value and accuracy, with exact 95\% Confidence Intervals. To put the performance of SARC-F in a wider context, we repeated the procedure for alternative case-finding tests described in the literature.

\section{Results}

The mean (SD) age of 73 patients $(78.1 \%$ women) was 77.8 (7.3) years. Of the entire group, 17 persons had sarcopenia based on the EWGSOP2 criteria, while 14 participants fulfilled the criteria for the SARC-F defined sarcopenia. Table 1, contains the characteristics of the study group. Overall, the included patients were multimorbid and taking multiple medications (median quantity of OTC preparations 2 , median quantity of prescription preparations 7.5) irrespective of their sarcopenia status. Cognitive impairment was present in $61.1 \%$, malnutrition or the risk of malnutrition were present in $32 \%$ and frailty based on Fried criteria in $29.2 \%$ of the participants. Patients affected by sarcopenia were older, and had lower educational status, and were affected with more diseases. They had lower BMI, including lower muscle and lower fat mass, lower mid-arm circumference and lower calf circumference. Patients with sarcopenia, in general, were characterized by lower self-reported physical activity, and worse values for physical performance and muscle strength indices (Table 1).

\section{Translation and cultural validation. Intra and interrater reproducibility}

First, in an initial group of 10 persons of wide educational range (age 79.7 (9.3) years, 50\% women) we assessed the ability by the participants to comprehend the questions correctly. We did that across different educational strata and across genders. Further, in another group of 20 participants we performed the assessment of the inter-rater and intra-rater agreement of each of the five items of the SARC-F test. Due to the fact that the answers were qualified into three levels, it was not possible to use the McNemar's test. Instead, to check for the degree of agreement, we used the simple, unadjusted, kappa statistic. Overall, we found that the.

inter-rater agreement was high (the kappa statistics ranging between 0.85 and 1.0) and that the intra-rater reproducibility of the questions was good (the kappa statistics ranging between 0.65 and 1.0). (Appendix 3).

The Cronbach's alpha test for internal consistency of questions (final study-group) was 0.82 for ability to lift and carry $10 \mathrm{lb}, 0.83$ for past year's history of falls, 0.76 for chair to bed transfer, 0.76 for climbing 10 stairs and 0.74 for walking across a room. Overall, the Cronbach's alpha was 0.82 . 
Table 1 Baseline Characteristics

\begin{tabular}{|c|c|c|c|c|c|c|c|}
\hline & \multirow[t]{2}{*}{ All $(n=73)$} & \multicolumn{2}{|l|}{ SARC-F } & \multirow[t]{2}{*}{$p$} & \multicolumn{2}{|c|}{ EWGSOP2 consenus statement } & \multirow[t]{2}{*}{$p$} \\
\hline & & Sarcopenia & No sarcopenia & & Sarcopenia & No sarcopenia & \\
\hline Age (years) & $77.8(7.3)$ & $77.6(8.3)$ & $77.9(7.1)$ & 0.91 & $82.1(9.2)$ & $76.6(6.1)$ & 0.006 \\
\hline Women $(\%)$ & 78.1 & 92.9 & 74.6 & 0.14 & 70.6 & 80.4 & 0.40 \\
\hline Education (years) & $14.0(3.2)$ & $12.0(3.2)$ & $14.4(3.1)$ & 0.01 & $13.3(3.3)$ & $14.2(3.2)$ & 0.34 \\
\hline Financial status & & & & 0.09 & & & 0.25 \\
\hline Income insufficient (\%) & 38.9 & 64.3 & 32.8 & & 29.4 & 41.8 & \\
\hline Living alone (\%) & 48.6 & 21.4 & 55.2 & 0.02 & 47.1 & 49.1 & 0.89 \\
\hline Walking assist device (\%) & 13.9 & 42.9 & 6.9 & 0.0005 & 29.4 & 9.1 & 0.04 \\
\hline Chronic diseases (number) & $4.7(2.6)$ & $6.1(2.8)$ & $4.3(2.5)$ & 0.02 & $5.8(2.4)$ & $4.3(2.6)$ & 0.04 \\
\hline Charlson Index (points) & $1(0-4)$ & $1.5(0-4)$ & $1(0-5)$ & 0.03 & $2(0-9)$ & $1(0-4)$ & 0.002 \\
\hline Medications (number) & $7.5(2-13)$ & $8(3-13)$ & $7(1-13)$ & 0.65 & $8(4-11)$ & $7(1-13)$ & 0.97 \\
\hline OTC-drugs (number) & $2(0-4)$ & $1.5(0-3)$ & $2(0-4)$ & 0.57 & $1(0-3)$ & $2(0-4)$ & 0.48 \\
\hline BMI $\left(\mathrm{kg} / \mathrm{m}^{2}\right)$ & $27.0(5.2)$ & $25.9(5.8)$ & $27.3(5.0)$ & 0.36 & $23.3(4.0)$ & $28.2(5.0)$ & 0.0004 \\
\hline WHR & $0.9(0.1)$ & $0.9(0.1)$ & $0.90(0.1)$ & 0.59 & $0.9(0.1)$ & $0.9(0.1)$ & 0.59 \\
\hline $\operatorname{ASMM}(\mathrm{kg})$ & $17.5(3.9)$ & $15.0(3.1)$ & $18.1(3.8)$ & 0.008 & $14.2(2.4)$ & $18.5(3.7)$ & $<0.0001$ \\
\hline Fat mass (kg) & $26.1(9.7)$ & $22.5(11.4)$ & $27.0(9.2)$ & 0.12 & $19.7(7.9)$ & $28.1(9.4)$ & 0.001 \\
\hline ASMM/BMI $\left[\mathrm{kg} /\left(\mathrm{kg} / \mathrm{m}^{2}\right)\right]$ & $0.7(0.1)$ & $0.6(0.1)$ & $0.7(0.1)$ & 0.03 & $0.6(0.1)$ & $0.7(0.1)$ & 0.19 \\
\hline $\operatorname{ASMM} / \mathrm{h}^{2}\left(\mathrm{~kg} / \mathrm{m}^{2}\right)$ & $6.8(1.2)$ & $6.4(1.3)$ & $6.9(1.1)$ & 0.15 & $5.9(0.8)$ & $7.1(1.1)$ & $<0.0001$ \\
\hline MAC (cm) & $29.9(4.2)$ & $28.7(5.8)$ & $30.1(3.7)$ & 0.37 & $26.7(3.8)$ & $30.8(3.8)$ & 0.0002 \\
\hline $\mathrm{CC}(\mathrm{cm})$ & $36.7(4.0)$ & $34.0(5.0)$ & $37.4(3.5)$ & 0.003 & $33.1(4.2)$ & $37.9(3.3)$ & $<0.0001$ \\
\hline Hand grip strength (kg) & $17.6(9.4)$ & $8.9(6.9)$ & $19.7(8.8)$ & $<0.0001$ & $11.7(7.1)$ & $19.4(9.4)$ & 0.003 \\
\hline Chair stand test (s) & $12.8(5.2)$ & $17.8(7.4)$ & $12.0(4.2)$ & 0.04 & $15.6(6.9)$ & $12.0(4.3)$ & 0.02 \\
\hline Gait speed $(\mathrm{m} / \mathrm{s})$ & $1.0(0.3)$ & $0.7(0.3)$ & $1.1(0.3)$ & $<0.0001$ & $0.8(0.3)$ & $1.1(0.3)$ & 0.002 \\
\hline SPPB (points) & $9.5(2.7)$ & $6.4(3.6)$ & $10.2(1.9)$ & 0.002 & $7.7(3.3)$ & $10.0(2.3)$ & 0.002 \\
\hline TUG (s) & $9.3(4.6)$ & $15.3(7.1)$ & $7.9(2.1)$ & 0.002 & $11.5(5.6)$ & $8.7(4.2)$ & 0.03 \\
\hline ADL (points) & $5.9(0.4)$ & $5.6(0.7)$ & $6.0(0.2)$ & 0.13 & $5.7(0.7)$ & $6.0(0.2)$ & 0.01 \\
\hline IADL (points) & $7.6(1.4)$ & $6.8(2.1)$ & $7.8(1.1)$ & 0.11 & $6.7(0.7)$ & $7.9(0.5)$ & 0.002 \\
\hline MoCA Test (points) & $22.6(4.8)$ & $21.3(4.5)$ & $22.9(4.9)$ & 0.27 & $18.2(5.9)$ & $23.9(3.6)$ & $<0.0001$ \\
\hline Cognitive impairment & & & & 0.52 & & & 0.0003 \\
\hline MCI & 37.5 & 35.7 & 37.3 & & 29.4 & 40.0 & \\
\hline Dementia & 23.6 & 35.7 & 22.0 & & 58.8 & 12.7 & \\
\hline GDS Test (points) & $3.7(2.7)$ & $5.1(3.3)$ & $3.4(2.5)$ & 0.03 & $3.7(2.9)$ & $3.7(2.7)$ & 0.99 \\
\hline \multicolumn{8}{|l|}{ Weight loss } \\
\hline Last 3 months (kg) & $0(0-5)$ & $1.3(0-5)$ & $0(0-5)$ & 0.04 & $0(0-5)$ & $0(0-5)$ & 0.38 \\
\hline Last 6 months (kg) & $0(0-10)$ & $3(0-10)$ & $0(0-10)$ & 0.03 & $0(0-5)$ & $0(0-10)$ & 0.65 \\
\hline Nutritional status & & & & 0.27 & & & 0.09 \\
\hline Risk of malnutrition (\%) & 27.8 & 42.9 & 24.1 & & 35.3 & 25.5 & \\
\hline Malnutrition (\%) & 4.2 & 7.1 & 3.5 & & 11.8 & 1.8 & \\
\hline Frailty (Fried; \%) & 29.2 & 85.7 & 15.3 & $<0.0001$ & 52.9 & 21.8 & 0.02 \\
\hline Frailty (Rockwood; \%) & 15.3 & 57.1 & 5.1 & $<0.0001$ & 41.2 & 7.3 & 0.001 \\
\hline PA & $\begin{array}{l}4343.8 \\
\quad(2242.7- \\
8951.6)\end{array}$ & $\begin{array}{l}3038.1 \\
\quad(1508.8- \\
17,484.2)\end{array}$ & $4352.0(2488.5-8470.0)$ & 0.11 & $\begin{array}{c}3696.0 \\
(1508.8- \\
5390.0)\end{array}$ & $4874.3(2346.0-9828.0)$ & 0.001 \\
\hline EQ-5 (points) & $0.8(0.1)$ & $0.7(0.1)$ & $0.9(0.1)$ & $<0.0001$ & $0.9(0.1)$ & $0.8(0.1)$ & 0.75 \\
\hline EQ-5 (\%) & $65.1(15.4)$ & $61.8(16.0)$ & $65.9(15.3)$ & 0.37 & $59.4(14.7)$ & $66.9(15.3)$ & 0.08 \\
\hline \multicolumn{8}{|l|}{ SARC-F } \\
\hline Strength & & & & $<0.0001$ & & & 0.008 \\
\hline None & 50.7 & 0.0 & 62.7 & & 23.5 & 58.9 & \\
\hline Some & 30.1 & 28.6 & 30.5 & & 41.2 & 26.8 & \\
\hline A lot of/unable to do & 19.2 & 71.4 & 6.8 & & 35.3 & 14.3 & \\
\hline
\end{tabular}


Table 1 (continued)

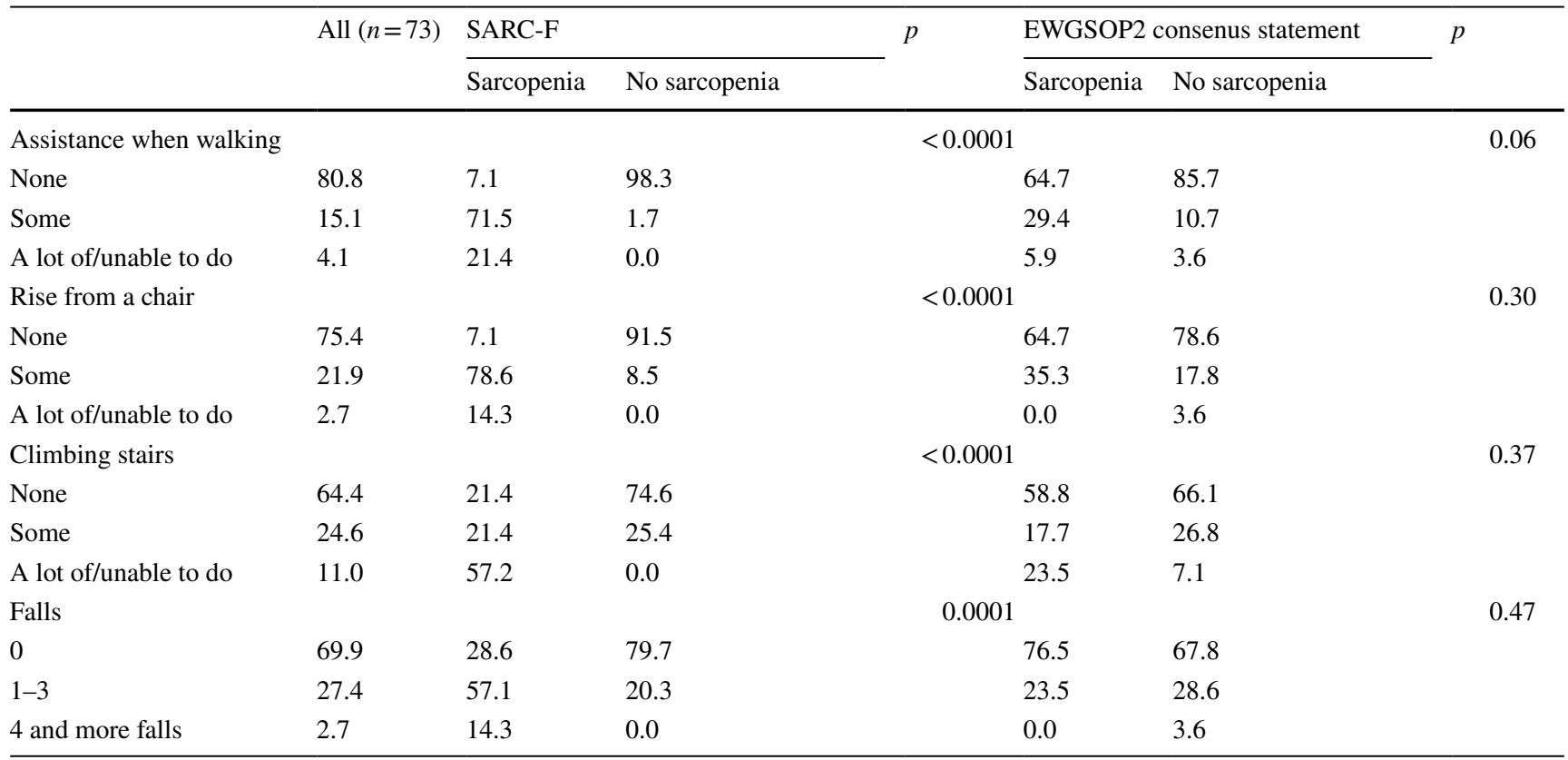

\section{Measures of clinical usefulness}

To assess the clinical usefulness of SARC-F to detect the cases of sarcopenia, we used, as standards the following definitions of sarcopenia: EWGSOP2, IWGS, FNIH, and SSCWD. The sensitivity and specificity of SARC-F was $35.3 \%$ and $85.7 \%$ for EWGSOP2, $38.5 \%$ and $85.0 \%$ for IWGS, 30.0\% and 82.5 for FNIH, 50.0\% and $86.9 \%$ for SSCWD. The corresponding PPV and NPV were $42.9 \%$ and $81.4 \%$ for EWGSOP2, 35.7\% and $86.4 \%$ for IWGS, $21.4 \%$ and $88.1 \%$ for FNIH, and 42.3 and $89.8 \%$ for SSCWD. The c-statistics for SARC-F were as follows: 0.64 for EWGSOP2, 0.60 for IWGS, 0.57 for FNIH, and 0.68 for SSCWD. The details are given in Table 2 .

\section{Sample-specific cut-off values for SARC-F}

Based on the ROC results for each of the sarcopenia definition used, we calculated sample-specific cut-off values of SARC-F. The Youden method-based cut-off with each sarcopenia standard as comparator was $\geq 5$, except for FNIH where it was $\geq 2$. The sensitivity of thus obtained cut-off against the FNIH as comparator was $60.0 \%$, specificity was $61.9 \%$, PPV was $20.0 \%$, NPV was $90.7 \%$. The sensitivity of this cut-off against the EWGSOP2 for was $35.3 \%$, specificity was $89.3 \%$, PPV was $50.0 \%$, NPV was $82.0 \%$. The corresponding values for the remaining comparator definitions of sarcopenia were not materially altered in comparison to standard SARC-F cut off. The details are given in Table 2.

\section{Other screening tools for sarcopenia}

To put the clinical validity of SARC-F in a broader context, we analyzed the clinical validity of other screening modalities for sarcopenia.

With an exception of mid-arm circumference alone against FNIH as standard, all additionally tested screening criteria demonstrated numerically better c-statistic and sensitivity compared to SARC-F. The results for specificity, PPV, NPV, and accuracy varied and are presented in Table 2.

\section{Discussion}

We performed a two-step, cultural and clinical validation of the Polish translation of the SARC-F questionnaire. We did that in the community-dwelling older persons, against the four commonly used definitions of sarcopenia. We used whole-body DXA scans to assess muscle mass.

SARC-F questionnaire was reproduceable both intra-rater (all kappa $\geq 0.82$ ) and inter-rater (all kappa $\geq 0.62$ ). The sensitivity and specificity of SARC-F against the EWGSOP2 was $35.3 \%$, and $85.7 \%$, respectively. We used the Youden method to obtain population-specific cut-off for SARC-F. This did not importantly improve the estimates of clinical validity.

SARC-F has been designed as a rapid screening tool for sarcopenia [10]. The SARC-F questionnaire has been widely translated and validated in specific populations [11-16, 22, $44,45]$. For some languages, the validation was performed 
Table 2 Sarcopenia Screening Tools validated against Sarcopenia Consensus Definitions

\begin{tabular}{|c|c|c|c|c|c|c|c|c|c|c|c|c|}
\hline \multirow[b]{2}{*}{ EWGSOP2 } & \multirow[b]{2}{*}{$17(23.3)$} & \multicolumn{2}{|c|}{ Sensitivity (\%) } & \multicolumn{2}{|c|}{ Specificity (\%) } & \multicolumn{2}{|c|}{ PPV (\%) } & \multicolumn{2}{|c|}{ NPV (\%) } & \multicolumn{2}{|l|}{ Accuracy } & \multirow[t]{2}{*}{ c-statistics } \\
\hline & & & & & & & & & & & & \\
\hline SARC-F & & 35.3 & $14.2-61.7$ & 85.7 & $73.8-93.6$ & 42.9 & $17.7-71.1$ & 81.4 & $69.1-90.3$ & 74.0 & $62.4-83.6$ & 0.64 \\
\hline SARC-F $\geq 5$ & & 35.3 & $14.2-61.7$ & 89.3 & $78.1-96.0$ & 50.0 & $21.1-78.9$ & 82.0 & $70.0-90.6$ & 76.7 & $65.4-85.8$ & \\
\hline SARC-CalF $\geq 11$ & & 41.2 & $18.4-67.1$ & 98.2 & $90.5-100.0$ & 87.5 & $47.4-99.7$ & 84.6 & $73.5-92.4$ & 84.9 & $74.6-92.2$ & 0.72 \\
\hline Ishii tool $\geq 136$ & & 88.2 & $63.6-98.5$ & 75.0 & $61.6-85.6$ & 51.7 & $32.5-70.6$ & 95.5 & $84.5-99.4$ & 78.1 & $66.9-86.9$ & 0.88 \\
\hline $\mathrm{CC}<32$ & & 41.2 & $18.4-67.1$ & 98.2 & $95.0-100.0$ & 87.5 & $47.4-99.7$ & 84.6 & $73.5-92.4$ & 84.9 & $74.6-92.2$ & 0.81 \\
\hline MAC $<29$ & & 70.6 & $44.0-89.7$ & 69.6 & $55.9-81.2$ & 41.4 & $23.5-61.1$ & 88.6 & $75.4-96.2$ & 69.9 & $58.0-80.0$ & 0.78 \\
\hline IWGS & $13(17.8)$ & & & & & & & & & & & \\
\hline SARC-F & & 38.5 & $13.9-68.4$ & 85.0 & $73.4-92.9$ & 35.7 & $12.8-64.9$ & 86.4 & $75.0-94.0$ & 76.7 & $65.4-85.8$ & 0.60 \\
\hline SARC-F $\geq 5$ & & 38.5 & $13.9-68.4$ & 88.3 & $77.4-95.2$ & 41.7 & $15.2-72.3$ & 86.9 & $75.8-94.2$ & 79.5 & $68.4-88.0$ & \\
\hline SARC-CalF $\geq 11$ & & 61.5 & $31.6-86.1$ & 88.3 & $77.4-95.2$ & 53.3 & $26.6-78.7$ & 91.4 & $81.0-97.1$ & 83.6 & $73.1-91.2$ & 0.83 \\
\hline Ishii tool $\geq 136$ & & 84.6 & $54.6-98.1$ & 70.0 & $56.8-81.2$ & 37.9 & $20.7-57.7$ & 95.5 & $84.5-99.4$ & 72.6 & $60.9-82.4$ & 0.78 \\
\hline $\mathrm{CC} \leq 35$ & & 76.9 & $46.2-95.0$ & 86.7 & $75.4-94.1$ & 55.6 & $30.8-78.5$ & 94.6 & $84.9-98.9$ & 84.9 & $74.6-92.2$ & 0.91 \\
\hline MAC $<28$ & & 84.6 & $54.6-98.1$ & 76.7 & $64.0-86.6$ & 44.0 & $24.4-65.1$ & 95.8 & $85.8-99.5$ & 78.1 & $66.9-86.9$ & 0.85 \\
\hline FNIH & $10(13.7)$ & & & & & & & & & & & \\
\hline SARC-F & & 30.0 & $6.7-65.3$ & 82.5 & $70.9-91.0$ & 21.4 & $4.7-50.8$ & 88.1 & $77.0-95.1$ & 75.3 & $63.9-84.7$ & 0.57 \\
\hline SARC-F $\geq 2$ & & 60.0 & $26.2-87.8$ & 61.9 & $48.8-73.9$ & 20.0 & $7.7-38.6$ & 90.7 & $77.9-97.4$ & 61.6 & $49.5-72.8$ & \\
\hline SARC-CalF $\geq 11$ & & 60.0 & $26.2-87.8$ & 65.1 & $52.0-76.7$ & 21.4 & $6.2-36.6$ & 91.1 & $78.8-97.5$ & 64.4 & $52.3-75.3$ & 0.69 \\
\hline Ishii tool $\geq 136$ & & 90.0 & $55.5-99.8$ & 68.3 & $55.3-79.4$ & 31.0 & $15.3-50.8$ & 97.7 & $88.0-99.9$ & 71.2 & $59.5-81.2$ & 0.81 \\
\hline $\mathrm{CC} \leq 37$ & & 80.0 & $44.4-97.5$ & 54.0 & $40.9-66.6$ & 21.6 & $9.8-38.2$ & 94.4 & $81.3-99.3$ & 57.5 & $45.4-69.0$ & 0.73 \\
\hline $\mathrm{MAC}<24$ & & 20.0 & $2.5-55.6$ & 92.1 & $82.4-97.4$ & 28.6 & $3.7-71.0$ & 87.9 & $77.5-94.6$ & 82.2 & $71.5-90.2$ & 0.55 \\
\hline SSCWD & $12(16.4)$ & & & & & & & & & & & \\
\hline SARC-F & & 50.0 & $21.1-78.9$ & 86.9 & $75.8-94.1$ & 42.3 & $17.7-71.1$ & 89.8 & $79.2-86.2$ & 80.8 & $69.9-89.1$ & 0.68 \\
\hline SARC-F $\geq 5$ & & 50.0 & $21.1-78.9$ & 90.2 & $79.8-96.3$ & 50.0 & $21.1-78.9$ & 92.0 & $79.8-96.3$ & 83.6 & $73.1-91.2$ & \\
\hline SARC-CalF $\geq 11$ & & 66.7 & $34.9-90.1$ & 100.0 & $94.1-100.0$ & 100.0 & $63.1-100.0$ & 93.9 & $85.0-98.3$ & 94.5 & $86.6-98.5$ & 0.81 \\
\hline Ishii tool $\geq 154$ & & 66.7 & $34.9-90.1$ & 86.9 & $75.8-94.2$ & 50.0 & $24.7-75.4$ & 93.0 & $83.0-98.1$ & 83.6 & $73.1-91.2$ & 0.79 \\
\hline $\mathrm{CC} \leq 32$ & & 66.7 & 34.9-90.1 & 98.4 & $91.2-100.0$ & 88.9 & $51.8-99.7$ & 93.8 & $84.8-98.3$ & 93.2 & $84.7-97.7$ & 0.91 \\
\hline MAC $<28$ & & 91.7 & $61.5-99.8$ & 77.1 & $64.5-86.9$ & 44.0 & $24.4-65.1$ & 97.9 & $88.9-100.0$ & 79.5 & $68.4-88.0$ & 0.90 \\
\hline
\end{tabular}

In addition to the SARC-F, we used the SARC-CalF including calf circumference on top of the SARC-F components as described by BarbosaSilva et al. [24] and a point scoring system designed by Ishii et al. [25] using sex, age, grip-strength, and calf-circumference

We also used midarm circumference and calf circumference as separate screening instruments. For the Ishii et al.'s index, the calf and midarm circumference, we used population-specific cut-off values based on Youden's Index. For the calculation SARC-CalF, on a scale from 0 to 20, we used the Youden Index-based population-specific cut off for calf circumference $(<32 \mathrm{~cm})$ with a weight of 10 . The cut-off for SARC-CalF was $\geq 11$

in several populations, sometimes yielding conflicting results $[12,13,46,47]$.

In Poland, a recent study of 67 community-dwelling persons $\geq 65$ years of age, the sensitivity of SARC-F was $92.9 \%$, and the specificity $98.1 \%$ [47]. This contrasts results of similar another Polish study, where the sensitivity of SARC-F was $41.2 \%$, specificity was $88.0 \%$ [46]. Both studies, used the bio-impedance weighting scale-measurement, however, the former one was performed in a slightly younger population $(69.5 \pm 4.0$ years vs. $74.5 \pm 6.9$ years $)$, which to some extend may have influenced the results. Based on DXA assessment, we show the sensitivity to be even smaller, with comparable specificity. A German validation study performed in 117 older subjects showed that the internal consistency of the German version of SARC-F was acceptable (the Cronbach alpha $=0.67$ ), with intra-rater repeatability of 0.90 and inter-rater repeatability of 0.93 . They estimated the sensitivity of SARC-F as $63 \%$, specificity as $47 \%$ and the c-statistic of 0.58 [48]. Our estimates of the inter-rater and intra-rater repeatability for test components were $>0.85$, and $>0.65$, respectively. The overall Cronbach alpha was 0.82 . The sensitivity was $35 \%$ and specificity $86 \%$. The c-statistic was 0.64 .

A number of studies, in persons of varied background including ethnicity, age, pathology were performed, yielding wide range of estimates of sensitivity, specificity, accuracy, and the ROC for SARC-F [20].

The largest study thus far was performed in 4000 older individuals from three populations of varied cultural background, demonstrated low sensitivity, but high specificity 
of SARC-F $(<10 \%,>94 \%$, gender-specific sensitivity and specificity, respectively). The authors of that report concluded that despite the low sensitivity and thanks to high specificity SARC-F may be used as a screening tool for sarcopenia at the community level [49].

SARC-F was employed in a range of pathologic settings. In the heart failure patients, the sensitivity and specificity were $52.5 \%$ and $96.2 \%$, respectively [44]. In hip fracture patients, sensitivity was $95 \%$ and specificity $57 \%$, [20] and in older orthopedic patients sensitivity was $47.4 \%$ and specificity $68.4 \%$ [50].

Disparate results of the psychometric characteristics of the SARC-F screening questionnaire demonstrated across sarcopenia studies, might be due to the varying diagnostic modalities used. As shown by Kim et al. in their study of 2099 community-dwelling older adults from the nationwide Korean Frailty and Aging Cohort Study (KFACS), sarcopenia prevalence varied from $7.9 \%$ if employing the chair stand test as a measure of muscle strength and ASM/height ${ }^{2}$ as a quantity unit for muscle mass, to $18.4 \%$ when handgrip strength and/or chair stand test and ASM/height ${ }^{2}$ results were examined [51].

In our study, we checked the diagnostic validity of the SARC-F against four definitions of sarcopenia (EWGSOP2, FNIH, IWGS, SSCWD expert guidelines). With grip strength as a measure of muscle strength, gait speed as a proxy of physical performance, and appendicular lean body mass checked with whole-body DXA scans, we showed an acceptable accuracy of the SARC-F for finding sarcopenia according to all the definitions tested. Additionally, we calculated the Youden's J statistic and constructed the population-specific cut-off values for SARC-F against the EWGSOP2, FNIH, IWGS and SSCWD sarcopenia working group diagnostic criteria. We showed a sensitivity of SARC-F ranged from 30 to $50 \%$ and specificity from 83 to $87 \%$, the results by and large in line with previously published data [18]. When setting the Polish population-specific cut off values for SARC-F questionnaire, we obtained better diagnostic properties when adjusting the threshold for suspected sarcopenia to five points for the EWGSOP2, IWGS and SSCWD, and two points for FNIH sarcopenia diagnostic consensus accordingly.

\section{The clinical performance of other screening tests}

We found the SARC-F tool to be fairly specific but with low sensitivity. To put that in a broader context, we checked the clinical validity of other sarcopenia screening tools with our study population-specific cut-offs. The tools included: an index designed by Ishii et al. [25], calf circumference alone, midarm circumference alone, SARC-CalF - an index based on SARC-F that incorporates calf circumference [24], and the SARC-F with the study population-specific cut-off values. We found that for most of those tools the sensitivity was numerically better than for SARC-F. We also noted that the very simple measures such as calf circumference, or the midarm circumference were characterized by best clinical performance.

\section{Limitations and strengths}

Our study needs to be considered in the context of its limitations. Our sample was moderate in size. However, its size was in line with what has been published thus far. We tested the SARC-F against an array of standards, where for the quantification of the muscle mass we used DXA. This may be an advantage over the studies that had used a bio-impedance based assessment of muscles.

\section{Conclusions and implications}

We present a validated Polish translation of the SARCF questionnaire. Although some other simple measures such as the mid-arm circumference or the calf circumference are at least of comparable value, SARC-F is more versatile, as it can be self-administered, assessed during a telephone interview, or used in subjects of varying bodybuild, or body-build affected by pathologies such as heart failure, liver failure, hypoalbuminemia etc. Our results indicate that its performance is better in ruling sarcopenia out than finding the cases.

Funding The project was funded by the research grant from the Jagiellonian University Medical College (funding: K/ZDS/007805).

Availability of data and materials Available upon reasonable request.Code availability Analysis was based on SAS Base procedures.

\section{Compliance with ethical standards}

Ethics approval Jagiellonian University Bioethics Committee approval no.: KBET 1072.6120.71.2018.

Human and Animal rights All the procedures in the study were in accordance with the ethical standards of the Jagiellonian University Ethical Committee and with the 1964 Helsinki Declaration (with amendments).

Consent for publication All authors have approved the manuscript for publication.

Consent to participate All participants signed an informed consent statement. 


\section{Conflict of interest None.}

Open Access This article is licensed under a Creative Commons Attribution 4.0 International License, which permits use, sharing, adaptation, distribution and reproduction in any medium or format, as long as you give appropriate credit to the original author(s) and the source, provide a link to the Creative Commons licence, and indicate if changes were made. The images or other third party material in this article are included in the article's Creative Commons licence, unless indicated otherwise in a credit line to the material. If material is not included in the article's Creative Commons licence and your intended use is not permitted by statutory regulation or exceeds the permitted use, you will need to obtain permission directly from the copyright holder. To view a copy of this licence, visit http://creativecommons.org/licenses/by/4.0/.

\section{References}

1. Mayhew AJ, Amog K, Phillips S et al (2019) The prevalence of sarcopenia in community-dwelling older adults, an exploration of differences between studies and within definitions: a systematic review and meta-analyses. Age Ageing 48:48-56. https://doi. org/10.1093/ageing/afy106

2. Pacifico J, Geerlings MAJ, Reijnierse EM et al (2020) Prevalence of sarcopenia as a comorbid disease: a systematic review and meta-analysis. Exp Gerontol 131:110801. https://doi. org/10.1016/j.exger.2019.110801

3. Beaudart C, McCloskey E, Bruyère $\mathrm{O}$ et al (2016) Sarcopenia in daily practice: assessment and management. BMC Geriatr 16:170. https://doi.org/10.1186/s12877-016-0349-4

4. Rizzoli R, Reginster JY, Arnal JF et al (2013) Quality of life in sarcopenia and frailty. Calcif Tissue Int 93:101-120. https://doi. org/10.1007/s00223-013-9758-y

5. Beaudart C, Rizzoli R, Bruyère O et al (2014) Sarcopenia: burden and challenges for public health. Arch Public Heal 72(1):45. https ://doi.org/10.1186/2049-3258-72-45

6. Cruz-Jentoft AJ, Bahat G, Bauer J et al (2019) Sarcopenia: Revised European consensus on definition and diagnosis. Age Ageing 48:16-31. https://doi.org/10.1093/ageing/afy169

7. Beckwée D, Delaere A, Aelbrecht S et al (2019) Exercise interventions for the prevention and treatment of sarcopenia. a systematic umbrella review. J Nutr Heal Aging 23:494-502

8. Robinson S, Granic A, Sayer AA (2019) Nutrition and muscle strength, as the key component of Sarcopenia : an overview of current evidence. Nutrients 11:2942

9. Bauer J, Morley JE, Schols AMWJ et al (2019) Sarcopenia: a time for action. An SCWD position paper. J Cachexia Sarcopenia Muscle 10:956-961. https://doi.org/10.1002/jcsm.12483

10. Malmstrom TK, Morley JE (2013) SARC-F: a simple questionnaire to rapidly diagnose sarcopenia. J Am Med Dir Assoc 14:531-532

11. Bahat G, Yilmaz O, Kılıç C et al (2018) Performance of SARC-F in regard to sarcopenia definitions, muscle mass and functional measures. J Nutr Health Aging 22:898-903

12. Sánchez-Rodríguez D, Marco E, Dávalos-Yerovi V et al (2019) Translation and validation of the spanish version of the SARC-F questionnaire to assess sarcopenia in older people. J Nutr Heal Aging 23:518-524. https://doi.org/10.1007/s12603-019-1204-z

13. Parra-Rodríguez L, Szlejf C, García-González A et al (2016) Cross-cultural adaptation and validation of the spanish-language version of the SARC-F to assess sarcopenia in mexican community-dwelling older adults. J Am Med Dir Assoc 17:1142-1146
14. Urzi F, Šimunič B, Buzan E (2017) Basis for Sarcopenia Screening With the SARC-CalF in Nursing Homes. J Am Med Dir Assoc 18:991.e5-991.e10

15. Kim S, Kim M, Won CW (2018) Validation of the Korean Version of the SARC-F questionnaire to assess Sarcopenia: Korean frailty and aging cohort study. J Am Med Dir Assoc 19:40-45.e1

16. Ida S, Murata K, Nakadachi D et al (2017) Development of a Japanese version of the SARC-F for diabetic patients: an examination of reliability and validity. Aging Clin Exp Res 29:935-942

17. Yang M, Lu J, Jiang J et al (2019) Comparison of four sarcopenia screening tools in nursing home residents. Aging Clin Exp Res 31:1481-1489. https://doi.org/10.1007/s40520-018-1083-x

18. Kim M, Won CW (2020) Sarcopenia in Korean communitydwelling adults aged 70 years and older : application of screening and diagnostic tools from the Asian working group for sarcopenia 2019 update. J Am Med Dir Assoc 21:752-758. https://doi. org/10.1016/j.jamda.2020.03.018

19. Ida S, Kaneko R, Imataka K et al (2020) Verification of the predictive validity for mortality of the SARC-F questionnaire based on a meta-analysis. Aging Clin Exp Res. https://doi.org/10.1007/ s40520-020-01585-1

20. Ida S, Kaneko R, Murata K (2018) SARC-F for screening of sarcopenia among older adults : a meta-analysis of screening test accuracy. J Am Med Dir Assoc 19:685-689. https://doi.org/10.1016/j. jamda.2018.04.001

21. Kera T, Kawai H, Hirano $\mathrm{H}$ et al (2020) Limitations of SARC-F in the diagnosis of sarcopenia in community-dwelling older adults. Arch Gerontol Geriatr 87:103959. https://doi.org/10.1016/j.archg er.2019.103959

22. Ha Y, Won C, Kim M et al (2020) SARC-F as a useful tool for screening sarcopenia in elderly patients with hip fractures. J Nutr Heal Aging 24:78-82

23. Bahat G, Yilmaz O, Oren MM et al (2018) Cross-cultural adaptation and validation of the SARC-F to assess sarcopenia: methodological report from European Union Geriatric medicine society sarcopenia special interest group. Eur Geriatr Med 9:23-28. https ://doi.org/10.1007/s41999-017-0003-5

24. Barbosa-Silva TG, Menezes AM, Bielemann RM et al (2016) Enhancing SARC-F: improving sarcopenia screening in the clinical practice. J Am Med Dir Assoc 17:1136-1141

25. Ishii S, Tanaka T, Shibasaki K et al (2014) Development of a simple screening test for sarcopenia in older adults. Geriatr Gerontol Int 14(Suppl 1):93-101

26. Leong DP, Teo KK, Rangarajan S et al (2016) Reference ranges of handgrip strength from 125,462 healthy adults in 21 countries: a prospective urban rural epidemiologic (PURE) study. J Cachexia Sarcopenia Muscle 7:535-546

27. Studenski S, Perera S, Wallace D et al (2003) Physical performance measures in the clinical setting. J Am Geriatr Soc $51: 314-322$

28. Mathias S, Nayak US, Isaacs B (1986) Balance in elderly patients: the "get-up and go" test. Arch Phys Med Rehabil 67:387-389

29. Guralnik JM, Ferrucci L, Pieper CF et al (2000) Lower extremity function and subsequent disability: consistency across studies, predictive models, and value of gait speed alone compared with the short physical performance battery. J Gerontol A Biol Sci Med Sci 55:M221-231

30. Magierska J, Magierski R, Fendler W et al (2012) Clinical application of the polish adaptation of the montreal cognitive assessment (MoCA) test in screening for cognitive impairment. Polish J Neurol Neurosurg 46:130-139

31. Yesavage JA (1988) Geriatric depression scale. Psychopharm Bull 24:709-711 
32. Cornelis E, Gorus E, Beyer I et al (2017) Early diagnosis of mild cognitive impairment and mild dementia through basic and instrumental activities of daily living: development of a new evaluation tool. PLoS Med 14:e1002250

33. Blair SN, Haskell WL, Ho P et al (1985) Assessment of habitual physical activity by a seven-day recall in a community survey and controlled experiments. Am J Epidemiol 122:794-804

34. Fried LP, Tangen CM, Walston J et al (2001) Frailty in older adults: evidence for a phenotype. J Gerontol A Biol Sci Med Sci 56:M146-156

35. Rockwood K, Song X, MacKnight C et al (2005) A global clinical measure of fitness and frailty in elderly people. CMAJ 173:489-495

36. MNA- Mini Nutritional Assessment. https://www.mna-elder ly.com/forms/mna_guide_english_sf.pdf Accessed 26 June 2020

37. EQ-5D-5L. https://euroqol.org/eq-5d-instruments/eq-5d-51-about / Accessed 26 June 2020

38. Charlson ME, Pompei P, Ales KL, MacKenzie CR (1987) A new method of classifying prognostic comorbidity in longitudinal studies: development and validation. J Chronic Dis 40:373-383

39. Anthropometry Procedures Manual. National Health and Nutrition Examination Survey (NHANES). https://www.cdc.gov/nchs/data/ nhanes/nhanes_07_08/manual_an.pdf accessed on-line on June 26, 2020

40. Dam TT, Peters KW, Fragala M et al (2014) An evidence-based comparison of operational criteria for the presence of sarcopenia. J Gerontol A Biol Sci Med Sci 69:584-590

41. Fielding RA, Vellas B, Evans WJ et al (2011) Sarcopenia: an undiagnosed condition in older adults. Current consensus definition: prevalence, etiology, and consequences. International working group on sarcopenia. J Am Med Dir Assoc 12:249-256

42. Morley JE, Abbatecola AM, Argiles JM et al (2011) Sarcopenia with limited mobility: an international consensus. J Am Med Dir Assoc 12:403-409

43. Fluss R, Faraggi D, Reiser B (2005) Estimation of the Youden Index and its associated cutoff point. Biom J 47:458-472
44. Zhao W, Lu M, Wang X, Guo Y (2020) The role of sarcopenia questionnaires in hospitalized patients with chronic heart failure. Aging Clin Exp Res. https://doi.org/10.1007/s40520-020-01561 -9.10.1007/s40520-020-01561-9

45. Kera T, Kawai H, Hirano H et al (2019) SARC-F: A validation study with community-dwelling older Japanese adults. Geriatr Gerontol Int 19:1172-1178. https://doi.org/10.1111/ggi.13768

46. Krzymińska-Siemaszko R, Tobis S, Lewandowicz M, Wieczorowska-Tobis $\mathrm{K}$ (2020) Comparison of four sarcopenia screening questionnaires in community-dwelling older adults from Poland using six sets of international diagnostic criteria of sarcopenia. PLoS ONE 15:e0231847. https://doi.org/10.1371/journ al.pone. 0231847

47. Zasadzka E, Trzmiel T, Pawlaczyk M (2020) Polish translation and validation of the SARC-F tool for the assessment of sarcopenia. Clin Interv Aging 15:567-574

48. Drey M, Ferrari U, Schraml M et al (2020) German version of SARC-F : translation, adaption, and validation. J Am Med Dir Assoc 21:747-751.e1. https://doi.org/10.1016/j.jamda .2019.12.011

49. Woo J, Leung J, Morley JE (2014) Validating the SARC-F: a suitable community screening tool for sarcopenia? J Am Med Dir Assoc 15:630-634. https://doi.org/10.1016/j.jamda.2014.04.021

50. Kurita N, Wakita T, Kamitani T et al (2019) SARC-F validation and SARC-F+EBM derivation in musculoskeletal disease: the SPSS-OK study. J Nutr Heal Aging 23:732-738

51. Kim M, Won CW (2019) Prevalence of sarcopenia in community-dwelling older adults using the de fi nition of the European working group on sarcopenia in older people 2: findings from the Korean Frailty and Aging Cohort Study. Age Ageing 48:910-916. https://doi.org/10.1093/ageing/afz091

Publisher's Note Springer Nature remains neutral with regard to jurisdictional claims in published maps and institutional affiliations.

\section{Affiliations}

\section{Karolina Piotrowicz ${ }^{1,2}$. Anna Głuszewska ${ }^{1,2}$. Joanna Czesak ${ }^{2,3}$. Małgorzata Fedyk-Łukasik ${ }^{1,2}$. Ewa Klimek ${ }^{1,2}$. Dolores Sánchez-Rodríguez ${ }^{4,5}$. Anna Skalska ${ }^{1,2}$ - Barbara Gryglewska ${ }^{1,2}$. Tomasz Grodzicki ${ }^{1,2}$ • Jerzy Gąsowski ${ }^{1,2}$ (1)}

1 Department of Internal Medicine and Gerontology, Faculty of Medicine, Medical College, Jagiellonian University, Ul. Jakubowskiego 2, 30-688 Kraków, Poland

2 Department of Internal Medicine and Geriatrics, University Hospital, Kraków, Poland

3 Department of Clinical Rehabilitation, University School of Physical Education, Kraków, Poland
4 Division of Public Health, Epidemiology and Health Economics, WHO Collaborating Centre for Public Health Aspects of Musculo-Skeletal Health and Ageing, University of Liège, Liège, Belgium

5 Geriatrics Department, Rehabilitation Research Group, Hospital Del Mar Medical Research Institute (IMIM), Universitat Pompeu Fabra, Barcelona, Spain 